\section{Endoscopic injections of Teflon to treat urinary incontinence in women}

Various forms of treatment have been developed to cure urinary stress incontinence in women. All surgical procedures aim to increase the urethral resistance to urine outflow and eventually to restore a normal angle between the proximal urethra and the bladder neck. ${ }^{1}$ Politano proposed using perineal injections of Teflon to treat incontinence in men after transurethral resection of the prostate and extended the technique to women. ${ }^{23} \mathrm{Heer}$ injected Teflon into the wall of the urethra to increase its length and closing pressure.$^{4}$ For the past three years we have used an endoscopic technique for giving injections of Teflon to women suffering from incontinence.

\section{Patients, methods, and results}

We treated 56 women aged 30-86 (mean 59) suffering from urinary stress incontinence. Twelve patients presented with cystoceles, four of whom had had an operation for this; 19 had undergone hysterectomy; four had undergone perineal amputation; and eight had had several operations to treat incontinence by classic vesicosuspension using sling procedures. Follow up ranged from three to 25 (mean 12) months.

An 8 French gauge polyethylene catheter ending in a needle was guided through the shaft of a regular cystoscope. The needle was inserted deep into the urethral wall, about $1 \mathrm{~cm}$ below the bladder neck. Teflon was injected using a small syringe with a metallic sheet and piston (Storz). About $3 \mathrm{ml}$ was injected at 1500,1800 , and 2100 (total around $10 \mathrm{ml}$ ). A bladder catheter was used for 24 hours afterwards, and patients stayed in hospital for about three days. Oral broad spectrum antibiotics were always prescribed for two weeks. Most injections were performed under general anaesthesia, but some were done under local anaesthesia or even sedation. When only partial improvement occurs Teflon injections can be repeated without difficulty after six to eight weeks, when the inflammation has resolved.

The table shows that $39(70 \%)$ of the 56 patients were completely cured, 29 after one, eight after two, and two after three injections. Cure was defined

Results of endoscopic Teflon injection for urinary incontinence in 56 women

\begin{tabular}{cccc}
\hline & \multicolumn{3}{c}{ No of patients showing: } \\
\cline { 2 - 4 } No of injections & Success & Improvement & Failure \\
\hline 1 & 29 & 5 & 2 \\
2 & 8 & 3 & 4 \\
3 & 2 & 1 & 1 \\
\hline 4 & $39(70 \%)$ & $9(16 \%)$ & $8(14 \%)$ \\
\hline
\end{tabular}

as the absence of leaking during the filling phase of a cystometrogram and was confirmed by questioning and examining the patient. Nine patients $(16 \%)$ showed substantial improvement in continence but complained of some urgency. Treatment failed in eight patients $(14 \%)$, of whom two had undergone perineal amputation followed by radiotherapy, one had interstitial cystitis, and one had neurological disorders. Complications were minimal. A few patients needed bladder catheters for two or three days. One woman with persistent urinary retention was treated by internal urethrotomy. Urethral burning and discomfort were experienced by most patients for one week.

\section{Comment}

Teflon paste is a suspension of fine polymer particles in glycerine and polysorbate. It has been used for years in surgery and otolaryngology and is an inert material that produces minimal inflammation. ${ }^{5}$ We initially used this technique only in patients who had had several previous operations without success. As the results were encouraging we used it for all patients eligible for classic operations. We noted minor complications such as occasional retention and bladder discomfort. Although no abscess developed, we prescribed broad spectrum antibiotics for two weeks.

Our main fear was of inducing obstruction of the bladder outlet, but measurement of urine flow after injection of Teflon showed an average decrease in rate of only $5 \mathrm{ml} / \mathrm{s}$, whereas a urethral pressure profile showed an appreciable increase in the functional length of the urethra. This technique has obvious advantages: hospital stay is shortened, obesity or previous surgical procedures do not prevent injection, and it does not hinder later open surgery.
'Stanton SL, Tanagho EA, eds. Surgery of female incontinence. Berlin, Heidelberg, New York: Springer-Verlag, 1980.

2 Politano VA, Small MP, Harper JM, Lynne CM. Periurethral Teflon injection for urinary incontinence. 7 Urol 1974;111:180-3.

${ }^{3}$ Politano VA. Periurethral polytetrafluorethylene injection for urinary incontinence. $\mathcal{F}$ Urol $1982 ; 127: 439-42$.

${ }^{4}$ Heer H. Die Behandlung der Härinkontinenz mit der Teflonpaste. Urol Int 1977;32:295-302.

5 Goff GE. Teflon injection for vocal cord paralysis. Arch Otolaryngol 1969; 90:98-102.

(Accepted 28 September 1983)

Department of Urology, University Clinics of Brussels, Erasme Hospital, Brussels, Belgium

C C SCHULMAN, MD, professor and chief of urology

J SIMON, MD, consultant urologist

E WESPES, MD, registrar

F GERMEAU, MD, consultant urologist

Correspondence to: Professor C C Schulman, Department of Urology, University Clinics of Brussels, Erasme Hospital, 808 Route de Lennik, 1070 Brussels, Belgium.

\section{Penicillamine induced myasthenia reactivated by gold}

We describe a patient whose penicillamine induced myasthenia was reactivated by gold. Her poor sulphoxidation state may have been related to the original induction of myasthenia.

\section{Case report}

A 52 year old woman developed seronegative rheumatoid arthritis in 1970 for which she was treated with indomethacin and aspirin until it deteriorated in 1976. Prednisolone $5 \mathrm{mg}$ daily was started but phased out after 18 months. In January 1977 she began penicillamine $125 \mathrm{mg}$ daily, gradually increasing to $500 \mathrm{mg}$ daily by August 1980 .

In June 1981 she began to feel increasingly tired. In November she had intermittent left ptosis and diplopia, variable weakness of neck flexors and triceps, with no bulbar or other weakness. Reflexes and sensation were intact. Edrophonium testing and tests for antiacetylcholine receptor and antinuclear antibodies gave positive results. Electromyography, plain chest radiography, and immunoglobulins IgA and IgG showed nothing abnormal. IgM concentration was $0 \cdot 4 \mathrm{~g} / 1$ (normal 0.6-3.0). Her HLA state was A1, A2, B12, DR2 DR4. Penicillamine induced myasthenia gravis was diagnosed and penicillamine stopped. After two months she had only occasional ptosis and after five months (May 1982) no myasthenic symptoms.

Her arthritis deteriorated, so fenclofenac $600 \mathrm{mg}$ twice daily was prescribed in March 1982. After two weeks a rash developed which resolved on withdrawing fenclofenac, recurring when she was rechallenged. Her myasthenia did not recur. In July 1982 she began sodium aurothiomalate $10 \mathrm{mg}$ weekly, increasing by $10 \mathrm{mg}$ a week for five weeks, when she developed a rash. Simultaneously her ptosis and diplopia reappeared. She required prednisolone (initially $10 \mathrm{mg}$ daily) for the rash, the dosage reducing over five months, and pyridostigmine (initially $60 \mathrm{mg}$ four times a day) for four months. Her myasthenia had resolved after six months.

\section{Comment}

No previous reports have described recurrence of penicillamine induced myasthenia when gold has been subsequently given. Our patient's myasthenia had virtually disappeared when she suffered a toxic reaction to fenclofenac, but this did not provoke recurrence of her myasthenia. Thus it is unlikely that the return of symptoms with gold was due to a non-specific upset. Since gold alone has never been reported to cause myasthenia, it probably aggravated a penicillamine induced defect.

The mechanism of action of penicillamine is not clear, but it affects the immune system and has induced several autoimmune disorders. It may directly stimulate oligoclonal B cell activity, upset the balance between $T$ helper and suppressor cells, or alter antigens by hapten formation. The sulphydryl group of penicillamine reduces disulphide bonds in several proteins. Acetylcholine receptor subunits also contain vulnerable disulphide bonds. Penicillamine binds, ${ }^{1}$ probably via these sulphydryl links, to subunits of electric fish acetylcholine receptor, reducing their affinity for acetylcholine. This may directly affect neuromuscular transmission, as in guinea pigs, where penicillamine 